\title{
Estrategias Didácticas y sus Dinámicas en el Tratamiento de los Trastornos Específicos del Lenguaje en el Estudiantado Chileno
}

\section{Didactic Strategies and their Dynamics in the Treatment of Specific Language Disorders in Chilean Students}

\author{
Braulio Navarro ${ }^{1, \star}$, Ninfa Álvarez ${ }^{2}$, Juan Carlos Beltrán ${ }^{3}$, Sebastián Peña ${ }^{4}$, Adolfo Guzmán ${ }^{5}$ \\ ${ }^{1}$ Universidad Bernardo O'Higgins, Chile \\ ${ }^{2}$ Liceo Francisco Hernández Ortiz Pizarro, Chile \\ ${ }^{3}$ Universidad de la Frontera, Chile \\ ${ }^{4}$ Universidad Austral de Chile, Chile \\ ${ }^{5}$ Universidad SEK, Chile
}

\section{DESCRIPTORES:}

Estrategias didácticas

Trastornos específicos del lenguaje

Necesidades

educativas especiales

Inclusión educativa

Calidad

\section{RESUMEN:}

La labor de los profesores(as) diferenciales y fonoaudiólogos(as) es importante en el proceso de aprendizaje de los(as) estudiantes con trastornos específicos del lenguaje, dicha labor debe estar asociada al conocimiento y evidencia vinculada al aula. Esta investigación describe las estrategias didácticas en el proceso de rehabilitación del trastorno específico del lenguaje utilizadas por las profesoras diferenciales y fonoaudiólogas en una escuela especial de lenguaje en Chile. La metodología utilizada corresponde a la cualitativa con un diseño de estudio de caso único. Los participantes de este estudio fueron: ocho profesoras diferenciales y dos fonoaudiólogas. Los principales hallazgos develan que las profesoras diferenciales y fonoaudiólogas centran sus estrategias didácticas en la planificación, evaluación, consejos y reuniones. Como conclusión se plantea que las estrategias didácticas utilizadas por las profesoras diferenciales se vinculan a elementos técnicos alejados de la reflexión crítica, es decir, que se asocian con la racionalidad técnica instrumental.

\section{KEYWORDS: \\ Didactic strategies \\ Specific language disorders \\ Special educational needs \\ Educational inclusion Quality}

\section{ABSTRACT:}

The work of special education teachers and speech pathologists is important in the learning process of students with specific language disorders, this work must be associated with knowledge and evidence linked to classroom. This research describes the didactic strategies of the rehabilitation process of specific language disorders used by special needs teachers and speech therapists in a special language school in Chile. The methodology used corresponds to the qualitative one with a single case study. The participants in this study were eight special needs teachers and two speech pathologists. The main findings reveal that special needs teachers and speech pathologists focus their teaching strategies on planning, evaluation, advice and meetings. In conclusion, it is stated that the didactic strategies used by the special needs teachers are linked to technical elements far from critical reflection, that is, they are associated with instrumental technical rationality.

Navarro, B., Álvarez, N., Beltrán, J. C., Peña, S. y Guzmán, A. (2021). Estrategias didácticas y sus dinámicas en el tratamiento de los trastornos específicos del lenguaje en el estudiantado chileno. REICE. Revista Iberoamericana sobre Calidad, Eficacia y Cambio en Educación, 19 (1), 23-38. https://doi.org/10.15366/reice2021.19.1.002 


\section{Antecedentes}

En Chile, la biblioteca del congreso nacional (BCN) manifiesta el Decreto Supremo №490 (1990) que establece la normativa de integrar estudiantes con discapacidad en establecimientos de educación regular. Con la llegada de la democracia a Chile y en el gobierno de transición de Patricio Aylwin Azócar, se establece la Ley de Integración Social de las Personas con discapacidad $N^{\circ} 19.284$, la ley decreta normas para la plena integración de las personas con discapacidad, y vela por el pleno ejercicio de los derechos constitucionales de los integrantes del estado chileno con necesidades educativas especiales (NEE) (BCN, 2010).

Las necesidades educativas especiales (NEE) son consideradas uno de los elementos fundamentales de la Reforma Educacional, lo cual da cuenta de la búsqueda de la equidad educativa. De este modo, se pretende asegurar el derecho a la educación a todas las personas desde el nivel parvulario. Además, establece la libertad de enseñanza como principio de la política y organización educativa del país a la luz de las políticas inclusivas.

La educación como elemento transversal de la sociedad se transforma en uno de los ejes principales de aplicación de la Ley de Integración, generándose a nivel de contexto educativo formal los programas de integración escolar (en adelante PIE), con el objetivo de abordar las problemáticas asociadas a la educación especial, y en específico a los niños y niñas con necesidades educativas especiales. Uno de los trastornos o patologías que forman parte de los programas PIE y que se vinculan al objeto de estudio de esta investigación, son los trastornos específicos del lenguaje (TEL).

Se entiende por TEL,

Un trastorno de la comunicación que interfiere en el desarrollo de las habilidades del lenguaje en niños que no tienen pérdida de audición o discapacidad intelectual. El trastorno especifico del lenguaje puede afectar el habla, la capacidad para escuchar, la lectura y la escritura de los niños. También se le conoce como trastorno del desarrollo del lenguaje, retraso del lenguaje o disfasia del desarrollo. (NIH, 2019, p. 1)

La evidencia científica nos plantea que el TEL es un trastorno que genera problemas en el desarrollo social e intelectual del niño, pero el tratamiento temprano de la problemática permite corregir esta patología (Coloma et al., 2015; Hernández, 2010; Keyserlingk, Castro y Carrasco, 2013; Mendoza, 2005; Schonhaut et al., 2007). Para Villanueva y otros (2008), los trastornos específicos del lenguaje se vinculan a la comunicación de característica oral, siendo esta problemática uno de los trastornos más comunes en niños y niñas en la primera etapa escolar. En la población de habla inglesa su prevalencia oscila entre el $2 \%$ y el $7 \%$.

De acuerdo con De Barbieri, Maggiolo y Alfaro (1999), la realidad chilena no es muy distinta a la de habla inglesa, el porcentaje en la primera etapa escolar de niños y niñas con TEL es de $4 \%$, siendo los varones la población más afectada. Además, los trastornos específicos del lenguaje en escolares tienen como principal característica la presencia de variaciones lingüísticas en la producción de palabras y situación de mayor complejidad como son ausencia o escasas vocalizaciones (Buiza, Rodríguez-Parra y Adrián, 2015; Hincapié et al., 2007; Mendoza, 2005) dichos trastornos también emergen y están presentes en la realidad chilena (Schonhaut et al., 2007; Valdivia et al., 2013).

López (2005) manifiesta que en la primera etapa escolar se pueden presentar dificultades en el lenguaje, que de no ser tratadas persisten durante todo el proceso educativo, teniendo un impacto en el desarrollo social y cognitivo del alumnado, lo anterior genera un obstáculo para el proceso de enseñanza aprendizaje que puede marcar el desarrollo a posteriori de los educandos.

Por tanto, ante la evidencia que nos manifiesta que el TEL es un problema que puede ser tratado y corregido en los niños y niñas que lo sufren, y en específico en el contexto escolar, resulta fundamental el trabajo de la educadora diferencial y fonoaudióloga, y su articulación que permite diagnosticar de forma prematura el problema, implementando estrategias didácticas remediales que atenúen o corrijan de forma definitiva el trastorno.

Es así como los niños(as) que presentan TEL requieren de un trabajo pedagógico orientado al correcto abordaje de su problemática, lo que requiere la convergencia de estrategias didácticas generadas desde la reflexión y el conocimiento disciplinar vinculado al trastorno lingüístico. Las estrategias didácticas son 
una serie de acciones que los docentes y la comunidad educativa utilizan con el objetivo de facilitar el proceso de enseñanza aprendizaje, pero para que las estrategias sean efectivas se requiere que los actores educativos se involucren en el proceso de rehabilitación.

Es en este contexto, que resulta fundamental el estudio de las estrategias didácticas utilizadas por las profesoras diferenciales en el proceso de rehabilitación de niños(as) con TEL que asisten a educación pre-escolar. En atención a ello, surge una serie de tensiones con relación a las prácticas pedagógicas en el trabajo del estudiantado con TEL y de las problemáticas que subyacen en la praxis del proceso de enseñanza aprendizaje del cual son parte las profesoras, padres, fonoaudiólogas, y el alumnado. Es a estas problemáticas y tensiones a donde se proyecta este estudio.

Si bien los procesos didácticos llevados a cabo por los docentes que trabajan con niños y niñas que presentan TEL forman parte del Programa de Integración, en la realidad estos programas están conformados por distintos profesionales, los cuales se articulan con la finalidad de generar transposición didáctica (Chevallard, 2009) de las estrategias didácticas, y favorecer el trabajo pedagógico. Es aquí, donde surgen ciertas suspicacias en relación con la aplicación y articulación del programa PIE por parte de los docentes, que se vinculan a problemas de gestión o a la presencia en el contexto educativo del estudio, de la racionalidad técnica instrumental.

Para Mansilla y Beltrán (2016), la racionalidad técnica instrumental se vincula a las estructuras de poder propias del paradigma curricular positivista, y de las teorías de enseñanza conductista, lo que da origen a un docente técnico instrumentalista. Estos docentes están lejos de las miradas curriculares interpretativas, y de teorías constructivistas, que promueven que los docentes sean prácticos reflexivos.

Es necesario manifestar que el estudio no busca explicar estas posibles problemáticas desde una lógica nomotética, más bien se busca conocer las convergencia de las estrategias didácticas desde miradas interpretativas que van construyendo una realidad que se transforma en un proceso consciente en acción y que nos da cuenta de que los datos arrojados por el ejercicio cualitativo tienen coherencia con la construcción de la realidad existente; es decir, el estudio centra su interés en la realidad construida por los docentes, realidad planteada como la construcción de sí mismo (Maturana y Varela, 2003).

En relación a la problemática, esta se genera de vivencias y de construcción histórica vívida en el contexto educativo por parte de los investigadores, y se vincula con la posible descontextualización de las estrategias didácticas en el trabajo con niños con TEL, todo esto provocado por distintas situaciones manifestadas en la práctica por los docentes; como son la falta de tiempo para planificar, la falta de espacio para generar la articulación metodológica informalidad en la articulación docente. Estos y otros elementos que obstaculizan las estrategias didácticas son corroborados por la evidencia empírica y la literatura internacional (Galve, 2002; Pomés y Sanhueza, 2013; Vega, 2009).

De acuerdo con lo planteado, se declara objeto de estudio las estrategias didácticas que utilizan los docentes en el trabajo con niños y niñas con TEL. Se entiende por estrategias didácticas, de acuerdo a Lucumi y González (2015),

Una acción de intervención que tiene como propósito alcanzar la formación de sujetos cognoscentes a través del desarrollo de habilidades y competencias que les permitan desenvolverse en un mundo cada vez más globalizado. Estos procesos de intervención deben pensarse desde el uso de estrategias, procesos y experiencias contextualizadas; es decir, es necesario tener en cuenta los intereses, habilidades y necesidades de formaciones de niños, niñas y jóvenes que asisten a los diferentes espacios de aprendizaje. (p. 112)

A modo general y en relación con la diversidad y el trabajo inclusivo asociado a necesidades educativas especiales como el TEL y a los contextos educativos, Cobeñas (2020) manifiesta que la escuela debe comprender que el alumnado es objeto del proceso educativo, es decir, las escuelas deben transformarse de acuerdo con las características del alumnado y no los niños y niñas cumplir con una serie de requisitos que los haga educables.

Por otra parte, Blanco (2006) manifiesta que en gran número los países incorporan leyes y principios vinculados a la educación para todos y todas, pero en la práctica pedagógica que pudiese estar asociada a las estrategias didácticas existen factores que excluyen y segregan a los alumnos y alumnas, generando problemas de equidad y calidad de las propuestas pedagógicas educativas 
Desde una mirada más específica, diferentes estudios (Blanco, 2007; Granada, Pomés y Sanhueza, 2013; Illán y Molina, 2011; Vega, 2009) que han implementado estrategias didácticas en niños que pertenecen a educación especial, plantean que la principales problemáticas presentes en el contexto educativo son las creencias y prejuicios de los profesores, y la estructura dominante que lleva a los docentes hacia métodos de enseñanza tradicionales que generan maestros y estudiantes autómatas, lejos de la anhelada educación critica, reflexiva y emancipadora.

Para Vega (2009), en su investigación cualitativa titulada "Integración de alumnos con necesidades educativas especiales", existe coherencia entre el discurso y las prácticas pedagógicas ejercidas por los profesores básicos y cuyo objetivo fue conocer las representaciones simbólicas que poseen los profesores en relación con el trabajo con niños con necesidades educativas especiales, se obtuvo como resultado que existe un discurso en la instituciones educativas en relación a la integración e inclusión educativa que no converge con la práctica pedagógica, como consecuencia de esto emergen prejuicios internalizados por el cuerpo docente y asumidos como parte del proceso educativo que obstaculizan el trabajo con niños y niñas con necesidades educativas especiales.

Es en este contexto que resulta fundamental estudiar las estrategias didácticas utilizadas por los docentes de programa PIE en el proceso de rehabilitación de niños(as) con TEL que asisten a educación pre- escolar, generando desde el método científico y desde lo sociolingüístico aportes al conocimiento con el objetivo de ampliar la mirada vinculada al objeto de investigación y a la problemática planteada.

Por tanto, se plantea desde la realidad educativa de una escuela especial de lenguaje en Chile y, a través de una investigación generada desde el paradigma interpretativo con un aproximación metodológica cualitativa, describir las estrategias didácticas utilizadas por las educadoras diferenciales y fonoaudiólogas en el proceso de rehabilitación de niños(as) con Trastornos Específicos de Lenguaje (TEL), pero de manera específica a) identificar las estrategias didácticas de aula enfocadas en el tratamiento de los Trastornos Específicos de Lenguaje; b) describir los diagnósticos fonoaudiológicos enfocados al tratamiento de los Trastornos específicos de lenguaje; c) describir la intervención de la profesora diferencial y fonoaudióloga de los Trastornos específicos de lenguaje; y d) comprender las dinámicas en ese espacio geo cultural, académico y simbólico en busca de contribuir al debate sobre estudios en educación especial, en términos de potenciar el carácter dinámico y relacional de este campo del conocimiento.

\section{Método}

Este estudio se enmarca en el enfoque interpretativo, dado que estudia el proceso de interpretación desde el punto de vista de las personas a fin de lograr la "comprensión" de los fenómenos (Guba y Lincoln, 1994). La metodología que se empleó para este estudio es cualitativa, planteándose como la acción humana cuyo fin último es la búsqueda del conocimiento, y que nace de las intersubjetividades de los grupos sociales. Para Taylor y Bogdan (1987) la investigación cualitativa tiene relación con la investigación que produce datos descriptivos de una conducta observable como es el caso de las conversaciones de las personas.

\section{Diseño}

El diseño de esta investigación se ajusta al estudio de caso, el cual según Stake (1999), es la persona o el grupo de personas que posee características tan particulares que le otorga el estatus de caso. En tal sentido, esta investigación se ajusta específicamente al estudio de caso único, según Stake (1999, p. 5), "nos interesan tanto por lo que tiene de único como por lo que tienen de común. Pretendemos comprenderlos". En este sentido, el caso lo constituye una escuela especial de lenguaje de la comuna de Valdivia, Chile.

\section{Categorías de análisis}

Las categorías corresponden a aquellos elementos de clasificación que suelen emplearse en la explicación teórica que emerge de los incidentes (Trinidad, Carrero y Soriano, 2006). De acuerdo con lo anterior, se plantean las categorías y su aproximación conceptual (cuadro 1). 


\section{Participantes}

Los participantes de la investigación fueron ocho profesoras diferenciales y dos fonoaudiólogas que trabajan con niños y niñas con una necesidad educativa especial transitoria (NEET), y están diagnosticados con trastorno especifico del lenguaje TEL.

Este estudio se focalizó en un grupo de profesoras que trabaja con niños con TEL y especialistas fonoaudiólogas encargadas de diagnosticar los trastornos y que son parte del programa de integración (PIE), las cuales deben articular su trabajo pedagógico con las especialistas fonoaudiólogas, las profesoras y fonoaudiólogas tienen edades que van entre los 30 y 40 años, y su experiencia en trabajo de aula es de entre 5 a 7 años (cuadro 2).

Cuadro 1

Categorías

\begin{tabular}{ll}
\hline \multicolumn{1}{c}{ Categoría } & \multicolumn{1}{c}{ Descripción } \\
\hline $\begin{array}{l}\text { Estrategias didác- } \\
\text { ticas }\end{array}$ & $\begin{array}{l}\text { Entendida como la serie de acciones que utiliza el profesor o profesora con la finalidad de } \\
\text { favorecer el proceso de enseñanza aprendizaje. }\end{array}$ \\
$\begin{array}{l}\text { Diagnóstico tras- } \\
\text { tornos específicos } \\
\text { de lenguaje }\end{array}$ & $\begin{array}{l}\text { Entendida como las estrategias diagnosticas utilizadas por las profesoras diferenciales y } \\
\text { las fonoaudiólogas en el tratamiento de los niños con TEL. }\end{array}$ \\
$\begin{array}{l}\text { Intervención profe- } \\
\text { sora diferencial }\end{array}$ & $\begin{array}{l}\text { Entendida como el conjunto de estrategias utilizadas por el profesor o profesora en el } \\
\text { tratamiento de los niños con TEL. }\end{array}$ \\
\hline
\end{tabular}

Nota. Elaboración propia.

Cuadro 2

Criterios de inclusión de los participantes

\begin{tabular}{ll}
\hline \multicolumn{1}{c}{ Criterio } & \multicolumn{1}{c}{ Descripción } \\
\hline $\begin{array}{l}\text { Género } \\
\begin{array}{l}\text { Rol o profesión que } \\
\text { desempeña }\end{array}\end{array}$ & Femenino y masculino \\
$\begin{array}{l}\text { Experiencias en la } \\
\text { docencia }\end{array}$ & $\mathrm{Al}$ menos 6 meses de trabajo docente en la escuela de lenguaje \\
\hline
\end{tabular}

Nota. Elaboración propia.

\section{Instrumento de obtención de información}

Para la recolección de datos se utilizó la entrevista semiestructurada a través de un guion, definida por Escribano (2008) como:

\section{La técnica cualitativa en donde se visualiza fuertemente que la indagación del mundo social parte y llega a una interacción humana. Entre todos los componentes que caracterizan a una entrevista existen dos que señalan claramente a ese lugar; su carácter dialógico y su estructura teórica. (p. 74)}

Las entrevistas fueron generadas de acuerdo con los objetivos de investigación y las categorías planteadas, las que se orientaron a las fonoaudiólogas y profesoras diferenciales vinculados al trabajo de niños con TEL. La primera etapa estuvo constituida por la generación de las preguntas que serían realizadas en el contexto de estudio, para luego proceder a la aprobación por parte de juicio de expertos.

En la segunda etapa se generó el pilotaje con el objetivo de analizar la aplicación de las entrevistas y corregir posibles problemas en el desarrollo del trabajo de campo oficial, mientras que en la tercera etapa se realizó la aplicación definitiva de las entrevistas, cuyos datos serán utilizados para la codificación abierta y codificación axial.

Para el estudio se generaron dos guiones de preguntas en relación con los objetivos del trabajo y la especialidad de las profesionales consultadas. La entrevista para las educadoras diferenciales estuvo constituida por once preguntas orientadas a identificar las estrategias didácticas de aula enfocadas en el tratamiento de los Trastornos Específicos de Lenguaje y describir la intervención de la profesora diferencial y fonoaudióloga de 
los Trastornos específicos de lenguaje. Las entrevistas a las fonoaudiólogas estuvieron constituidas por nueve preguntas que se orientaron a describir los diagnósticos fonoaudiológicos enfocados al tratamiento de los Trastornos específicos de lenguaje y describir la intervención junto a la profesora diferencial.

\section{Trabajo de campo}

Entrada: El primer contacto con los sujetos de estudio se efectuó vía telefónica para solicitar una entrevista con el director del centro educativo. La entrada al campo se realizó a través de una entrevista con el director, a quien se le hizo entrega de una carta formal de solicitud de autorización para realizar el estudio y requerir acceso a información de los participantes, además se indicó el propósito del estudio.

Desarrollo: La recolección de información se llevó a cabo en los horarios acordados con las docentes y fonoaudiólogas(os), a través de la entrevista semi-estructurada. Las entrevistas se aplicaron en espacios proporcionados por el establecimiento. La hora acordada se flexibilizó, considerando el rol activo del grupo objetivo frente a las tareas diarias del establecimiento, de tal manera que no tuviera interferencias con sus actividades programadas.

Salida: La salida del campo se realizó en el momento en que los datos comenzaron a repetirse, punto en el cual, ya no es posible encontrar información relevante que pueda generar aportes al estudio. En relación con lo expuesto, Glaser y Strauss (1967) emplean el término "saturación teórica" para aludir al momento del proceso de trabajo de campo en el que los datos comienzan a repetirse y no se logran nuevos hallazgos importantes.

\section{Criterios éticos}

En relación con la dimensión ética del estudio en esta investigación se utilizó el consentimiento informado. Al respecto Taylor y Bogdan (1987), manifiestan que subyace al ingreso al campo de estudio un contrato explícito que debe ser respetado por parte del investigador, lo primero es proteger el anonimato de los participantes, segundo el investigador debe evitar interferir en las actividades de los investigados. Para ello, se hizo entrega de una carta formal a los participantes del estudio, en la cual se les invitó a participar de forma voluntaria de una entrevista semiestructurada. Además, se consideraron los elementos propuestos por Helsinky (2013): a) Consentimiento informado, b) Confidencialidad y anonimato, y c) Retorno social de la información obtenida.

\section{Análisis de datos}

El análisis de los datos que emanaron de la entrevista semiestructurada, los cuales se realizaron con el programa Atlas ti 7.5. Para el proceso de codificación de los datos se incorporaron elementos de la teoría fundamentada, como la codificación abierta y axial. La codificación abierta, permitió descubrir, nombrar y desarrollar los conceptos, se debe abrir el texto y exponer los pensamientos, ideas y significados contenidos en él (Strauss y Corbin, 2002), y de esta forma, se levantaron categorías y subcategorías de manera inductiva. Durante la codificación abierta los datos se desagregaron en partes discretas, y se examinaron minuciosamente y compararon en busca de similitudes y diferencias (Strauss y Corbin, 2002), para luego realizar familias de códigos. En este contexto se fue codificando los relatos de manera minuciosa, al amparo del objeto de estudio. En este proceso de análisis de datos, se tuvo en cuenta cuatro elementos (i) La identificación del código (etiqueta), (ii) La definición del código, (iii) La descripción global de lo que significa el código, y (iv) La cita donde se ve representado el código.

Además, en reiteradas ocasiones los investigadores volvieron al sujeto, cuando la información resultó ser ambigua o superficial, a fin de indagar en profundidad respecto de los significados. Para ello se crearon memos, donde se establecieron preguntas y reflexiones. En tal sentido, Corbin, Bowers, Charmaz y Clarke (2009), Glaser y Strauss (1967), y Morse y otros (2009) señalan que los memos contribuyen a incrementar el nivel conceptual de la investigación y permite al investigador pensar y reflexionar más allá de los incidentes simples que lo llevan a identificar temas y patrones en los datos. Esto ayudó aportar datos novedosos, los cuales, a su vez, permitieron a los investigadores relacionar las categorías y subcategorías con mayor claridad.

Esto último, permitió llevar acabo la codificación axial, donde se relacionaron las categorías con sus subcategorías, con la finalidad de entregar explicaciones más precisas y completas sobre el fenómeno (Strauss y Corbin, 2002). Este proceso permitió depurar y diferenciar las categorías y subcategorías derivadas de la codificación abierta. 
Para Flick (2007), las categorías axiales se enriquecen por su ajuste con el mayor número de pasajes posible, para luego elaborar las relaciones entre categorías y subcategorías. Los procesos de codificación abierta y axial se realizaron, mediante la utilización del Método Comparativo Constante (MCC), "este procedimiento se convierte en un método de comparación constante cuando los intérpretes cuidan de comparar los códigos una y otra vez con los códigos y las clasificaciones obtenidas” (Flick, 2007, p. 248). Además, este procedimiento permitió triangular los datos de forma continua. Posteriormente, en el apartado de los resultados se describen las categorías y se presentan los resultados por subcategorías y, a su vez, se realiza el proceso de interpretación de estos. Todo el proceso de análisis expuesto se realizó entre investigadores que ejecutaron el estudio para evitar los sesgos y desviaciones. Finalmente, se discuten los resultados por categoría, a la luz de la teoría.

\section{Resultados}

A continuación, se exponen los resultados y la discusión de estos, por categorías y sus códigos correspondientes, a la luz de la teoría (figura 1).

Figura 1

Estrategias didácticas

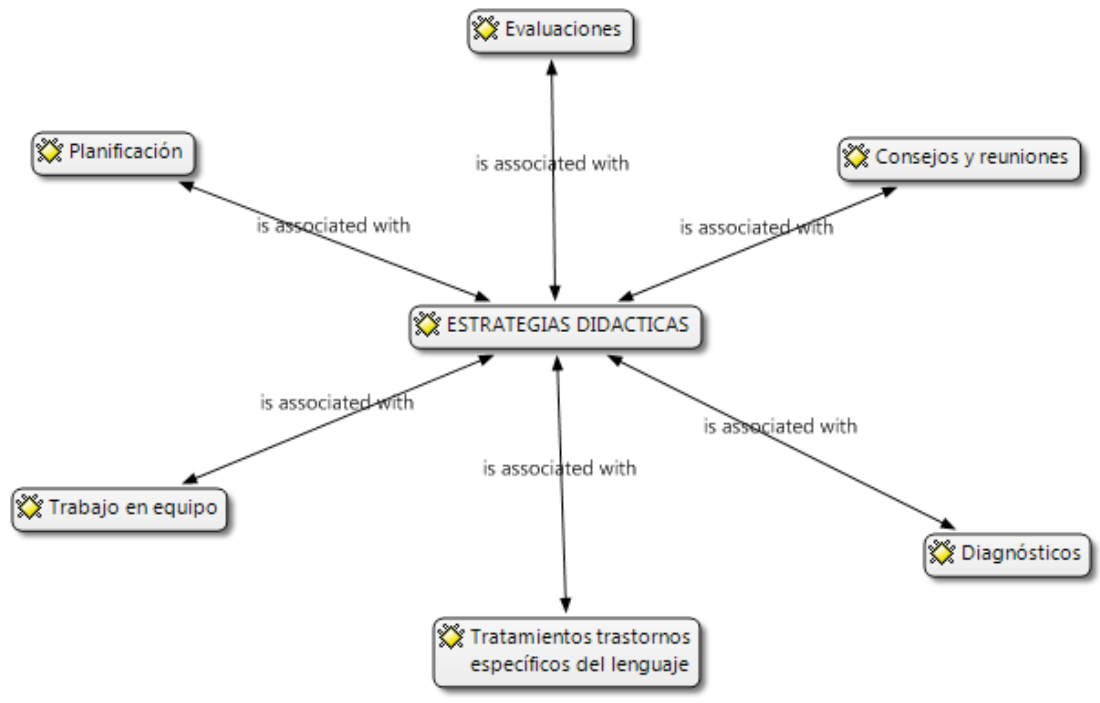

Nota. Elaboración propia.

\subsection{Categoría 1: Estrategias didácticas}

En esta categoría se describen las estrategias didácticas utilizadas por las profesoras diferenciales en el tratamiento de los niños con TEL. De esta categoría se despliegan las siguientes subcategorías: planificación, evaluaciones, consejo, reuniones, diagnóstico, tratamiento trastornos específicos del lenguaje y trabajo en equipo.

De acuerdo al relato y análisis de contenido vinculado a la planificación, la profesora plantea que las estrategias didácticas utilizadas se asocian a planificación de las intervenciones basadas en las bases curriculares de educación parvularia, estas planificaciones son generadas en consejos técnicos formados por la directora del establecimiento, jefe de UTP, profesoras y fonoaudiólogas, esto se respalda en la siguiente textualidad:

Las estrategias de planificación la organizamos a través de consejos técnicos con reunión técnica (UTP, directora, fonos), consejo técnico (fonos, directora, UTP, profesoras). (E3:2)

Además, las profesoras diferenciales en relación a las estrategias didácticas y las evaluaciones, manifiestan que se generan evaluaciones asociadas a la evaluación diagnóstica con el objetivo de analizar el proceso de tratamiento específico de los niños con TEL: 
Las estrategias de evaluación son elaboradas en consejos, reuniones, planificadas con la fono. (E4:1)

En este contexto, emergieron los consejos y reuniones, las profesoras manifiestan en relación a las estrategias didácticas utilizadas en el trabajo con niños y niñas con TEL que en la institución educativa se genera una serie de planificaciones periódicas para el trabajo de las bases curriculares de educación parvularia que orienta la aplicación de estrategias didácticas vinculadas al equipo de gestión, pero, en específico a la educadora diferencial y fonoaudiólogas. Esto se refleja en el siguiente relato:

El trabajo se organiza en reuniones y consejos a través de planificaciones mensuales y semanales basadas en las bases curriculares de educación parvularia. Reuniones entre fonoaudiólogay profesora diferencial. Al inicio se aplica prueba diagnóstica y semestralmente hay evaluaciones trimestrales. (E5:2)

Respecto a los diagnósticos se aprecia que las estrategias didácticas están vinculadas a planificaciones semanales y mensuales para nivelar los procesos de enseñanza aprendizaje y las técnicas de calificación de los procesos de trabajo académico. Lo expuesto, se aprecia en la siguiente declaración:

Las estrategias son diagnósticas y se organiza con planificaciones semanales y mensuales para trabajar los aprendizajes del nivel (bases curriculares de educación parvularia) y a través de reuniones con la fonoaudióloga para trabajar PEI. Se evalúa trimestral con pruebas y se completan las libretas de los niños con sus observaciones respecto de la prueba aplicada. (E6:1)

En el tratamiento del TEL, se visualiza la presencia del equipo de trabajo conformado por la educadora diferencial, asistente de educación y fonoaudióloga que trabajan de forma individual con los niños y niñas en sesiones de aproximadamente treinta minutos. Lo anterior, se respalda en la siguiente textualidad:

Las estrategias son parte del equipo de trabajo que se encarga de trabajar el TEL de los niños en forma individual. La fonoaudióloga saca a tres niños por sesión de 30 minutos y profesora especialista trabaja de la misma forma, pero en sala, mientras tanto el resto del grupo trabaja con tía asistente. (E6:6)

Referente al trabajo en equipo la estrategia didáctica manifestada consiste en reuniones del equipo de trabajo para enfocar el tratamiento específico de los niños y niñas con TEL, lo cual se manifiesta en la siguiente narración:

Las estrategias son de trabajo en equipo con la Fonoaudióloga, UTP, educadora diferencial, más el apoyo de la asistente. Se realizan reuniones para enfocar el tratamiento especifico. (E1:3)

Figura 2

Diagnósticos trastornos específicos del lenguaje

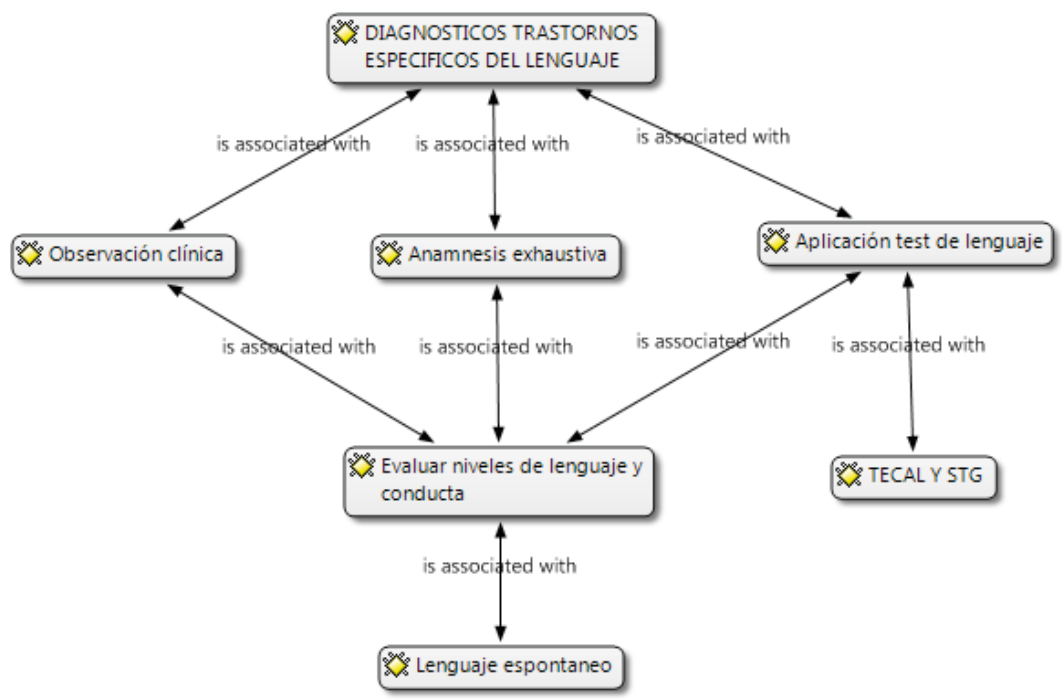

Nota. Elaboración propia. 


\subsection{Categoría 2: Diagnósticos trastornos específicos del lenguaje}

En esta categoría se describen las estrategias didácticas asociadas al diagnóstico fonoaudiológicos del TEL utilizadas por las profesoras diferenciales. Forman parte de esta categoría los códigos: observación clínica, lenguaje espontáneo, evaluar niveles lenguaje y conducta, aplicación de test de lenguaje, TECAL y STSG y anamnesis exhaustiva.

La subcategoría observación clínica, las profesoras plantean que las estrategias utilizada se asocian a la observación individual de los(as) estudiantes con la finalidad de generar intervenciones individuales más eficaces; además, las profesoras manifiestan que la observación debe estar orientada al lenguaje espontáneo, asociado a la evaluación de los niveles de lenguaje y conducta, ya que esto permite un diagnóstico más exhaustivo asociado a órganos fono auriculares (OFA), test de articulación a la repetición (TAR), test para evaluar procesos de simplificación fonológica (TEPROSIF), test exploratorio de la gramática española (STSG) y test para la comprensión auditiva del lenguaje (TECAL). Esto se respalda en la siguiente textualidad:

Se debe realizar una observación clínica del menor sobre su lenguaje espontáneo, conducta. Además de aplicar test para evaluar los niveles de lenguaje tanto expresivo como comprensivamente: OFA, anamnesis, TAR, TEPROSIF, STSG (expresivo, comprensivo), TECAL, por nombrar algunos. (E1:4)

Vinculado a la aplicación de test lenguaje, se observa que para diagnosticar es necesario aplicar test de lenguaje, siendo los más utilizados el TECAL y STSG. Además, se plantea que los diagnósticos más recurrentes son los trastornos específicos de lenguaje expresivo y mixto, lo que se respalda en la siguiente textualidad:

Los diagnósticos más recurrentes en el aula son el TEL mixto y TEL expresivo. (E4:3)

Por último, las profesoras plantean que es necesario generar una anamnesis exhaustiva, orientada a comprender la problemática desde el aula hasta la familia. Esto se respalda en el siguiente relato:

Es necesario una anamnesis exhaustiva para saber de dónde se origina el problema, ya que este puede vincularse a la familia. (E6:5)

Figura 3

Intervención profesora diferencial

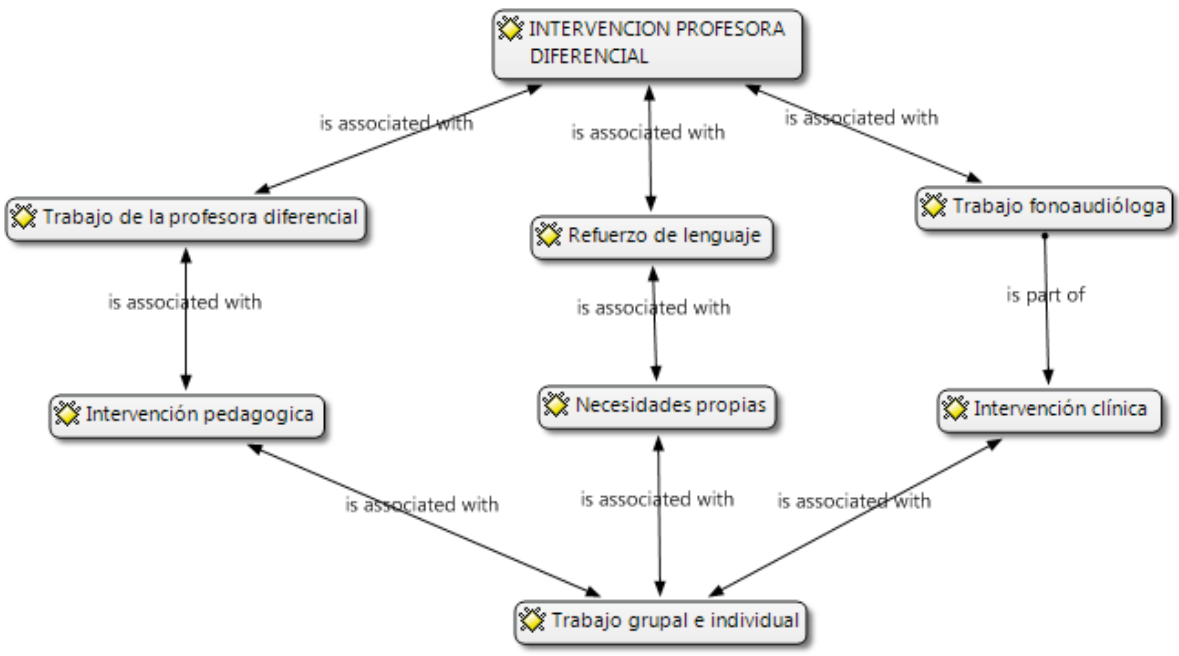

Nota. Elaboración propia.

\subsection{Categoría 3: Intervención profesora diferencial}

En esta categoría se describen las estrategias didácticas asociadas a la intervención de los trastornos específicos del lenguaje que utilizan las profesoras diferenciales y fonoaudiólogas. De la categoría antes 
descrita, se despliegan los siguientes códigos: Trabajo de la profesora diferencial, trabajo fonoaudióloga, refuerzo de lenguaje, intervención clínica, trabajo pedagógico, necesidades propias y trabajo grupal e individual.

Referente al trabajo de la profesora diferencial y trabajo fonoaudióloga, las profesoras plantean que se articulan con el objetivo de generar refuerzos en el lenguaje.

Además, las profesoras manifiestan que ellas se encargan del trabajo pedagógico y las fonoaudiólogas se encargan de la intervención clínica. De esta forma, gestionan el trabajo mancomunado desde las necesidades propias del alumnado, generando un trabajo metodológico grupal e individual, lo cual se manifiesta en las siguientes declaraciones: El plan específico es individual acorde a la necesidad que presenta cada niño, además de actividades grupales que permite reforzar el lenguaje (E5: 8). Otro entrevistado narra: "Se trabaja de a grupos de tres alumnos media hora una vez por semana con fonoaudióloga y en forma individual 10 minutos una vez a la semana con profesora especialista” (E6: 8). Asimismo, se aprecia el trabajo en individual:

El equipo de trabajo se encarga de trabajar el TEL de los niños en forma individual. La fo-
noaudióloga saca a tres niños por sesión de 30 minutos y profesora especialista trabaja de la
misma forma, pero en sala, mientras tanto el resto del grupo trabaja con tía asistente. (E7:8)
Sumado a lo anterior, se evidencia el trabajo en equipo entre la profesora diferencial y la
fonoaudióloga, vinculado con la práctica pedagógica en el aula: Es un trabajo a la par entre
profesoray fonoaudióloga. Además, se realiza un plan mensualy semanal haciendo clases en
aula en torno a una temática pedagógica. (E8:8)

Por último, las profesoras expresan que se trabaja un plan específico de forma individual y en grupos de tres alumnos, media hora una vez por semana con fonoaudióloga y, en forma individual 10 minutos una vez a la semana, con profesora especialista. También, explican que los equipos están compuestos por fonoaudióloga, UTP, educadora diferencial, asistente y que, desde la directora, sostenedora, profesoras, auxiliares de aseo, asistentes de la educación tienen que ver con la intervención. Ahora bien, profundizan en que se organizan por medio de consejos técnicos y reuniones, que la diversidad se aborda por medio de planificaciones mensuales y semanales, trabajos en la sala de recursos y se planifica el PIE con la fonoaudióloga.

De acuerdo a lo planteado, las profesoras centran el abordaje tanto de la evaluación como de la terapia del TEL básicamente en los niveles del lenguaje, olvidando parte de los aspectos cognitivos. Desde esta perspectiva, para los resultados del presente estudio resulta fundamental conocer las características cognitivas de los niños con TEL para robustecer el abordaje terapéutico, considerando la diversidad de elementos presentes en el proceso de enseñanza y aprendizaje, situando las estrategias didácticas en el contexto del conocimiento de los alumnos y alumnas.

\section{Discusión}

Respecto de la categoría estrategias didácticas, se evidencia que las profesoras diferenciales utilizan las estrategias didácticas, asociadas en el trabajo con niños y niñas con TEL. Las educadoras plantean que se basan en seis elementos asociados a la: planificación, evaluaciones, consejo, reuniones, diagnóstico, tratamiento trastornos específicos del lenguaje y trabajo en equipo e individual. Para Lucumi y González (2015), las estrategias didácticas son definidas como una serie de acciones de intervención cuyo objetivo es la formación cognitiva a través del desarrollo de habilidades y competencias. Estos procesos deben generarse desde el uso de estrategias y experiencias contextualizadas. Además, para que las estrategias sean efectivas es preciso considerar el interés, habilidades y necesidades de formación de los individuos.

Las estrategias didácticas observadas están más orientadas a una enseñanza tradicionalista, pues no se vinculan al ser situado históricamente a la enacción como proceso que otorga sentido a la acción humana, y en este caso a la acción docente, por lo tanto, desde la perspectiva de las profesoras diferenciales los resultados de esta investigación tienen relación con lo planteado por Mansilla y Beltrán (2016), vinculado a que las profesoras actúan bajo racionalidad técnica instrumental. En este contexto las estrategias didácticas deben responder a un aprendizaje situado, dado que implica la construcción del conocimiento, 
y es concebido como una práctica en la experiencia, por lo que aprender implica involucrarse en una comunidad de práctica (Driscoll, 2005).

En palabras de Díaz-Barriga (2003), la generación de conocimiento emerge en el contexto, dicho contexto está influenciado por la cultura y paradigmas de base de los actores sociales, por lo cual, ante esta realidad las estrategias didácticas deben emerger de la práctica y experimentación pedagógica, de esta forma se logra que los currículos escolares emerjan de los alumnos y se rompan las barreras de transposición didáctica flexibilizando los procesos educativos y favoreciendo los procesos de enseñanza aprendizaje (Alba, Sánchez y Zubillaga del Río, 2011).

Referente a la categoría diagnósticos trastornos específicos del lenguaje, que utilizan los docentes en el trabajo con niños y niñas con TEL, estos se vinculan a la observación clínica, lenguaje espontaneo, evaluar niveles lenguaje y conducta, aplicación de test de lenguaje: TECAL y STSG y anamnesis exhaustiva.

Para Moreno-Flagge (2013), la intervención metodológica de los niños y niñas con TEL, se inicia generando una anamnesis clínica diagnóstica de los niños y niñas, por otra parte, contar con antecedente de su etapa de gestación y posibles agentes familiares con antecedentes de TEL o enfermedades del tracto auditivo resulta fundamental.

Moreno-Flagge (2013) plantea que la observación sistemática del niño o niña y su entorno es importante y que la estimulación del vocabulario a través de preguntas que promuevan el desarrollo del lenguaje permite detectar alteraciones en el léxico o aparato auditivo. Se recomienda la aplicación de cuestionarios para padres y maestros y algunas pruebas específicas de lenguaje (test de vocabulario de Boston), el test de desarrollo de Bayley y la evaluación de inteligencia por el psicólogo, cuando sea necesaria. En este caso, se evidencia la presencia de la aplicación de test de lenguaje: TECAL y STSG. Respecto al primero, evalúa la comprensión del lenguaje en secciones de vocabulario, morfología y sintaxis en niños entre 3 y 6 años 11 meses (Maggiolo et al., 2014; Pavez, 2005).

En cuanto al test STSG, evalúa lenguaje expresivo y receptivo desde los 3 a los 6 años 11 meses. Está compuesta por una sub-prueba receptiva, que consta de 23 ítems donde el niño debe reconocer el dibujo que representa a la oración que se dice y una sub-prueba expresiva compuesta por 23 ítems en los que el niño debe repetir la oración correcta de acuerdo con la imagen que la representa (Lepe-Martínez et al., 2017; Pavez, 2005).

Sumado a lo anterior se evidencia que cobra relevancia realizar una anamnesis exhaustiva, orientada a comprender la problemática desde el aula hasta la familia. Al respecto, Rodríguez y Rodríguez (1999) señalan que, el estudio clínico denominado anamnesis que se ejecuta a través de una entrevista permite comprender y conocer elementos básicos del paciente junto a su historia de vida que se pudiesen vincula a su trastorno especifico del lenguaje.

Por último, Moreno-Flagge (2013) menciona que terminada la anamnesis clínica diagnóstica que nos permite excluir elementos externos, se debe centrar la intervención en la recepción y emisión como posible trastorno mixto. Si la problemática es la emisión el niño y niña tiene una comprensión normal, pero si el problema emerge en la recepción esta afecta en la comprensión y manifestación de palabras. De acuerdo a lo anterior, las profesoras diferenciales generan un diagnóstico adecuado de los niños y niñas con TEL, ya que este se logra a través de la observación y análisis individual de la problemática, lo que permite individualizar las alteraciones generando una metodología de acuerdo a las necesidades propias del estudiantado con TEL, pero no se manifiesta la existencia de estrategias didácticas situadas en el diagnóstico pedagógico que genera el punto de inicio de los tratamientos.

En la categoría intervención profesora diferencial, se evidencia el trabajo con niños y niñas con TEL, a partir de la articulación entre la profesora diferencial y la fonoaudióloga con el objetivo de generar refuerzo de lenguaje, intervención clínica, trabajo pedagógico, necesidades propias y trabajo grupal e individual. Al respecto, Moreno-Flagge (2013) plantea que el tratamiento del TEL es generado por terapeutas del lenguaje que en un inicio usaban técnicas de repetición de palabras u/o ejercicios gramaticales. Con el tiempo esto ha evolucionado a la comunicación natural y espontánea que facilite la socialización, por lo cual, la metodología de trabajo habitual es en grupos de niños y niñas con TEL.

Sumado a lo anterior, es relevante tomar en cuenta la interacción del niño(a) desde una perspectiva situada, lo que permite relacionar el desarrollo del lenguaje con la interacción entre la actividad mental y 
el medio social del niño, por lo que el lenguaje será adquirido en situaciones concretas de uso y de real intercambio comunicativo (Espinoza et al., 2004). En este contexto, aprendizaje tiene su origen en un entorno social y que el lenguaje capacita a los humanos en el desarrollo de funciones mentales superiores tales como la memoria intencional y la atención voluntaria, la planificación, el aprendizaje y el pensamiento racional.

Desde planteamientos de la teoría sociocultural, el aprendizaje es un proceso beneficioso de transformación cognitiva y social que se da en un contexto colaborativo, es decir, aprendemos al observar y participar con otros individuos y por mediación de artefactos culturales en actividades dirigidas hacia una meta (Antón, 2010). Es decir, que el niño(a) aprende a través de la interacción y colaboración con otros sujetos sobre la base de la mediación de instrumentos, mecanismos y formas de enseñanza que están presentes en actividades culturales, sociales e históricas.

Por otra parte, las profesoras diferenciales y fonoaudiólogas manifiestan estrategias didácticas de intervención orientadas a lo individual y buscan comprometer a la comunidad educativa en esta labor, pero no se manifiesta la existencia de técnicas específicas de trabajo vinculado al conocimiento disciplinario puro, basando la estrategia didáctica en lo clínico y pedagógico.

\section{Conclusiones}

Las conclusiones del estudio se presentan de acuerdo con el objetivo de describir las estrategias didácticas en el proceso de rehabilitación del trastorno específico del lenguaje utilizadas por las profesoras diferenciales y fonoaudiólogas de una escuela especial en Chile. Con el fin de alcanzar este propósito se plantean las categorías de análisis y el objetivo específico en torno a los cuales se presentan las conclusiones.

En relación con la categoría estrategias didácticas utilizadas por las profesoras diferenciales y fonoaudiólogas y el objetivo de identificar las estrategias didácticas de aula enfocadas en el tratamiento de los Trastornos Específicos de Lenguaje, éstas se vinculan a la racionalidad instrumental manifestada por elementos técnicos como la evaluación, planificación y articulación docente. No se evidencia manejo teórico del concepto estrategias didáctica, por lo cual, tampoco existe claridad en cuales aplican.

En relación con la categoría diagnósticos de los Trastornos específicos de lenguaje y el objetivo de describir los diagnósticos fonoaudiológicos enfocados al tratamiento de los Trastornos específicos de lenguaje (TEL), se constata que las fonoaudiólogas expresan una serie de test aplicados a los niños y niñas que se logra a través de la observación y análisis individual de la problemática, lo que permite individualizar las alteraciones, generando metodologías de acuerdo a las necesidades propias del estudiantado con TEL, pero no se manifiesta la presencia de estrategias didácticas desde su construcción teórica y aplicación práctica.

En relación con la categoría intervención de la profesora diferencial en el tratamiento de los Trastornos específicos de lenguaje (TEL) y el objetivo de describir la intervención de la profesora diferencial y fonoaudióloga de los Trastornos específicos de lenguaje, las intervenciones de la profesora se orientan a lo individual y buscan comprometer a la comunidad educativa en esta labor, pero no se manifiesta la existencia de técnicas específicas de trabajo vinculado al conocimiento disciplinario puro, situado desde estrategias didácticas en su construcción teórica y aplicación práctica.

Por último, y en atención al objetivo comprender las dinámicas en ese espacio geo cultural, académico y simbólico en busca de contribuir al debate sobre estudios en educación especial, en términos de potenciar el carácter dinámico y relacional de este campo del conocimiento, se constata la existencia de un gran consenso entre las profesoras, pues se asume que las estrategias didácticas se vinculan exclusivamente a elementos técnicos y prácticos, en correspondencia se manifiesta que las pedagogas se alejan de perspectivas curriculares interpretativas y de teorías constructivistas, que originan docentes prácticos reflexivos, asumiendo que las estrategias didácticas se vinculan solo a la racionalidad técnica instrumental.

En relación con las limitaciones que se presentaron durante esta investigación se puede manifestar problemáticas vinculadas a la escasa evidencia empírica afín al objeto de estudio y a la realidad chilena situada en el contexto educativo y en la práctica pedagógica, las distintas complicaciones para acceder a los sujetos de estudio y, por tanto, a los datos que permitiesen la generación de la investigación y saturación 
de la información. Respecto a la información producida por este estudio, atendiendo a la contingencia nacional y lo relevante que representa para el contexto educativo nacional y a los nuevos desafíos educativos que emprenderá el país, es del todo procedente seguir la línea investigativa que motivó este trabajo, desde una perspectiva más amplia respecto a las problemáticas vinculadas al trabajo con niños y niñas con necesidades educativas especiales, diversificando los estudios desde paradigmas de investigación mixtos que permitan enriquecer la mirada investigativa .

En una línea similar al punto precedente, se recomienda para el diseño de nuevas investigaciones en el área, considerar un contexto más amplio, es decir, aumentar la cantidad de participantes para poder extrapolar los resultados, además, es fundamental ampliar la comprensión de la problemática, por lo cual, emerge la necesidad de realizar una mirada cuantitativa de la realidad que permita enriquecer la visión del tema.

\section{Referencias}

Asociación Médica Mundial. (2013). Declaración de Helsinki de la AMM. Principios éticos para las investigaciones médicas en seres humanos. Asociación Médica Mundial. https://www.wma.net/es/policies-post/declaracion-de-helsinki-de-la-amm-principios-eticos-para-las-investigaciones-medicas-en-seres-humanos/

Alba, P., Sánchez, J. y Zubillaga del Río, A. (2011). Diseño universal para el aprendizaje. Pautas para su introducción en el currículo. https://www.educadua.es/doc/dua/

Antón, M. (2010). Aportaciones de la teoría sociocultural al estudio de la adquisición del español como segunda lengua. Resla, 23, 9-30.

Blanco, C. (2006). La equidad y la inclusión social: Uno de los desafíos de la educación y la escuela hoy. REICE. Revista Iberoamericana sobre Calidad, Eficacia y Cambio en Educación, 4(3),1-15.

Blanco, R. (2007). Hacia una escuela para todosy con todos. Boletín del proyecto principal de educación para América Latina y el Caribe. OREALC/UNESCO.

BCN. (2010). Ley de integración social de las personas con discapacidad $N^{\circ} 19.284$. https://www.leychile.cl/Navegar?idNorma=30651

Buiza, J. J., Rodríguez-Parra, M. J. y Adrián, J. A. (2015). Trastorno específico del lenguaje: Marcadores psicolingüísticos en semántica y pragmática en niños españoles. Anales de Psicología, 31(3), 879-889. https://doi.org/10.6018/analesps.31.3.180091

Chevallard, Y. (2009). La transposición didáctica-del saber sabio al saber enseñado. Aique.

Cobeñas, P. (2020). Exclusión educativa de personas con discapacidad: Un problema pedagógico. REICE. Revista Iberoamericana sobre Calidad, Eficacia y Cambio en Educación, 18(1), 65-81. https://doi.org/10.15366/reice2020.18.1.004

Coloma, C. J., Silva, M., Palma, S. y Holtheuer, C. (2015). Reading comprehension in children with specific language impairment: An exploratory study of linguistic and decoding skills. Psykhe, 24(2), 1-8. https://doi.org/10.7764/psykhe.24.2.763

De Barbieri Z., Maggiolo, M. y Alfaro S. (1999). Trastornos de la comunicación oral en niños que asisten a control de salud en un consultorio de atención primaria. Revista Chilena de Pediatría, 70(1), 36-40.

Díaz, F. y Hernández, G. (2006). Estrategias docentes para un aprendizaje significativo. McGraw-Hill.

Díaz-Barriga, F. (2003). Cognición situada y estrategias para el aprendizaje significativo. Revista Electrónica de Investigación Educativa, 5(2), 105-117.

Driscoll, M. (2005). Chapter: Situated congnition. Psychology of learning for instructión. Pearson.

Escribano, O. (2008). El proceso de investigación social cualitativo. Editorial Prometeo 
Espinoza, A. González, A., Hermosilla, S. y Tapia, S. (2004). Conciencia fonológica y desarrollo del conocimiento del lenguaje escrito en niños con trastorno específico del lenguaje expresivo. Universidad de Chile.

Flick, U. (2007). Introducción a la investigación cualitativa. Morata.

Galve, J. y Trallero, M. (Ed.). (2002). Adaptaciones curriculares. Editorial CEPE.

Glaser, B. y Strauss, A. (1967). The discovery of grounded theory: Strategies for qualitative research. Aldine. https://doi.org/10.1097/00006199-196807000-00014

Granada, M., Pomés, M. P. y Sanhueza, S. (2013). Actitud de los profesores hacia la inclusión educativa. Papeles de Trabajo, 25, 51-59.

Guba, E. y Lincoln, Y. (1994). Competing paradigms in qualitative research. En N. K. Denzin e Y. S. Lincoln (Eds.), Handbook of qualitative research (pp. 105-117). Sage.

Hernández, I. (2010). Aproximación a la neuropsicología y trastornos del lenguaje. Boletin de Linguistica, 22(34), 133-138.

Hincapié, L., Giraldo, M., Castro, R., Lopera, F., Pineda, D. y Lopera, E. (2007). Propiedades lingüísticas de los trastornos específicos del desarrollo del lenguaje. Revista Latinoamericana de Psicología, 39(1), 47-61.

Illán, N. y Molina, J. (2011). Integración curricular: Respuesta al reto de educar en y desde la diversidad. Educar em Revista, 41, 17-40. https://doi.org/10.1590/S0104-40602011000300003

Keyserlingk, L., Castro, P. J. y Carrasco, J. (2013). Teorías subjetivas de profesionales de escuelas de lenguaje en chile sobre el trastorno específico del lenguaje. Revista CEFAC, 15, 873-883. https://doi.org/10.1590/S1516-18462013005000039

Lepe-Martínez, N., Pérez-Salas, C., Rojas-Barahona, C. y Ramos-Galarza, C. (2017). Funciones ejecutivas en niños preescolares con y sin trastorno del lenguaje. Revista Ecuatoriana de Neurología, 26(3), 197-202.

López, S. (2005). La prevención de dificultades del lenguaje oral en el marco escolar: Evaluación e intervención temprana. Pensamiento Psicológico, 5, 73-83.

Lucumi, P. y González, M. (2015). El ambiente digital en la comunicación, la actitud y las estrategias pedagógicas utilizadas por docentes. Revista TED: Tecné, Episteme y Didaxis, 37, 109-129. https://doi.org/10.17227/01213814.37ted109.129

Maggiolo, M., Varela, V., Arancibia, C. y Ruiz, F. (2014). Dificultades de lenguaje en niños preescolares con antecedente de prematuridad extrema. Revista Chilena de Pediatría, 85(3), 319-327. https://doi.org/10.4067/S0370-41062014000300008

Maturana, H. y Varela, F. (2003). El árbol del conocimiento: Las bases biológicas del entendimiento humano. Grupo Editorial Lumen.

Mendoza, F. (2005). Trastorno específico del lenguaje: Concepto, clasificaciones y criterios de identificación. Revista Neurol, 41(1), S51-S56. https://doi.org/10.33588/rn.41S01.2005317

Moreno-Flagge, N. (2013). Trastornos del lenguaje. Diagnóstico y tratamiento. Revista Neurol, 57(1), 85-94. https://doi.org/10.33588/rn.57S01.2013248

Morse, J., Stern, P., Corbin, J., Bowers, B., Charmaz, K. y Clarke, A. (2009). Developing grounded theory. The second generation. Left Coast Press.

NIH. (2019). Trastornos especificos del lenguaje. Departamento de salud y servicios humanos de los EE. UU. Institutos Nacionales de la Salud.

Pavez, M. (2005). Test exploratorio de gramática española de A.Toronto. Universidad Católica de Chile.

Rodríguez, P. y Rodríguez, L. (1999). Principios técnicos para realizar la anamnesis en el paciente adulto. Revista Cubana de Medicina General Integral,15(4), 409-414. 
Schonhaut, L., Maggiolo, M., De barbieri, Z., Rojas, P. y Salgado, A. M. (2007). Dificultades de lenguaje en preescolares: Concordancia entre el test TEPSI y la evaluación fonoaudiológica. Revista Chilena de Pediatría, 78(4), 369-375. https://doi.org/10.4067/S0370-41062007000400004

Stake, R. E. (1999). Investigación con estudio de casos. Morata.

Strauss, A. L. y Corbin, J. (2002). Bases de la investigación cualitativa: Técnicas y procedimientos para desarrollar la teoría fundada. Editorial Universidad de Antioquia.

Taylor, S. J. y Bogdan, R. (1987). Introducción a los métodos cualitativos de investigación. La búsqueda de significados. Paidós.

Valdivia, I., Abadal, G. V., Gárate, E., Regal, N., Castillo, G., Sáez, Z. M. y Lozano, T. (2013). Factores biológicos asociados al retardo primario del lenguaje en niños menores de cinco años. Revista Cubana de Pediatría, 85(4), 466475.

Vega, A. (2009). Integración de alumnos con necesidades educativas especiales. ¿Existe coherencia entre el discurso y las prácticas pedagógicas ejercidas por los profesores básicos? Estudios Pedagógicos, 35(2), 189-202. https://doi.org/10.4067/S0718-07052009000200011

Villanueva, P., Babieri, Z., Palomino, M. H. y Palomino, H. (2008). Alta prevalencia de trastorno específico de lenguaje en isla Robinson Crusoe y probable efecto fundador. Revista Médica de Chile, 136, 186-192. https://doi.org/10.4067/S0034-98872008000200007

\section{Breve CV de los/as autores/as}

\section{Braulio Navarro}

Doctor en Ciencias de la Educación, Profesor de Educación Física deportes y recreación, Magister en motricidad infantil. Investigador del centro de investigación educativa (CIE) del doctorado en Educación de la Universidad Bernardo O’Higgins de Santiago, Chile. El doctor Braulio Navarro ha desarrollado sus líneas de investigación vinculadas a la inclusión y políticas públicas asociadas a la diversidad, la formación inicial docente y las neurociencias y aprendizaje. En la academia ha sostenido una extensa trayectoria como docentes en el contexto escolar público, particular subvencionado y particular en instituciones chilenas. En el contexto universitario centra su interés en la pedagogía e investigación educativa. Email: 002ademir@gmail.com

ORCID ID: https://orcid.org/0000-0002-3676-6222

\section{Ninfa Álvarez}

Educadora Diferencial, Especialista en Problemas de Aprendizaje. Magíster en Educación Diferencial con mención en Trastornos de la comunicación, audición y lenguaje. Docente Liceo Francisco Hernández Ortiz Pizarro, en el programa de integración escolar, Calbuco (Chile). La investigadora centra su trabajo en el contexto escolar siendo parte del Método Matte orientado al trabajo con necesidades educativas especiales en primero básico. Ha trabajo en diversas instituciones educativas dentro de las que se encuentran Jardín infantil particular Amardas, Puerto Montt (Técnico en párvulos), Colegio Innovasur en Puerto Montt. (Educadora de diferencial). Colegio los Conquistadores, Valdivia. (Educadora Diferencial). Escuela Alemania, Valdivia. Email: ninfa.alvarez09@gmail.com

ORCID ID: https://orcid.org/0000-0003-0797-8696

\section{Juan Carlos Beltrán}

Profesor Educación General Básica. Licenciatura en Educación, por la Universidad Central de Chile. Magister en Ciencias de la Educación Mención Currículum y Evaluación, por la Universidad Mayor, Temuco, Chile. Dr. en Ciencias de la Educación, por la Universidad de la Frontera, Temuco, Chile. Dr. en Educación por la Universidad de Sao Paulo, Brasil. Académico de la Universidad de La Frontera. Facultad de Educa- 
ción, Ciencias Sociales y Humanidades: Departamento de Educación. Líneas de investigación: Didáctica, currículum y educación intercultural. Email: jcarlosumayor@gmail.com

ORCID ID: https://orcid.org/0000-0003-4614-0650

\section{Sebastián Peña}

Doctor en Ciencias de la Educación por la Universidad de la Frontera, Máster en Innovación, Evaluación y Calidad de la Educación Física por la Universidad Autónoma de Madrid, Profesor de Educación Física por la Universidad de Los Lagos (Chile). Actualmente profesor Titular de la Universidad Austral de Chile, Instituto de Ciencias de la Educación, Facultad de Filosofía y Humanidades. Profesor invitado del Doctorado de Educación por la Universidad SEK-Chile. Sus líneas de investigación se centran en la formación inicial docente, el conocimiento teórico conceptual en educación física y las neurociencias vinculadas al aprendizaje. Email: sebap988@hotmail.com

ORCID: https://orcid.org/0000-0002-5438-0868

\section{Adolfo Guzmán Muñoz}

Doctor en Filosofía y en Estudios de la Comunicación, Master en artes y comunicación, ambos posgrados en la Universidad de Texas de Austin, Estados Unidos. Licenciado en Comunicación Social de la Universidad de Chile. Además de haber trabajado como director de planificación en agencias internacionales de comunicación, en la academia ha sostenido una extensa trayectoria en diversos cargos directivos en instituciones chilenas y como docente e investigador en la línea de neurociencia, comunicación, semiótica y educación. Email: adolfo.guzman@zonavirtual.uisek.cl

ORCID ID: https://orcid.org/0000-0002-5201-0433 